a number of applications in various fields, enabling this area of research with a rich future. Examples cited by Toma include supramolecular chemistry used to form very sensitive and powerful surfaceenhanced Raman spectroscopy (SERS) molecular probes. In one example, gold nanorods combined with antibodies with supramolecular chemistry yielded a way to identify cancer cells using SERS, wherein there was a clear difference in the Raman spectra of healthy and cancerous cells. This was also used in photothermal cancer therapy. In another example, silver nanoparticles were functionalized with imidazoles in poly-2-vinylpyridine electrospun hydrogels for use as bactericidal-fungicidal dressings for treating wounds. Some other examples mentioned by Toma included nanosensors for security, photoelectrochemical cells, superparamagnetic nanoparticles combined with supramolecular chemistry for biocatalysis and for capturing cells, and vanadium oxide-supramolecular porphyrin nanocomposites used as an ethanol fuel sensor.

\section{Materials Education Needs to Address Global Challenges}

The Ouroborus, an ancient symbol depicting a serpent swallowing its own tail and thus forming a circle, can be used to represent the importance of uniting scientific work performed at the microand nanoscale with humankind and the universe as a whole, said S. Mascarenhas (Univ. of São Paulo, São Carlos) in his keynote address on the challenges facing materials education in the 21st century. As the global population is expected to double by the end of the century and as a result of global climate changes, he said there will be an increased demand for energy, water, food, health care, and sustainability. Mascarenhas emphasized the crucial role that nanotechnology, and specifically bionanotechnology, could play in overcoming these challenges through the development of nanomaterials such as catalysts, hydrogen and biomass systems, nano-agro materials, and bionano-pharmaceuticals. In order to tackle these global challenges, efforts are needed to create a new educational structure in materials science and nanotechnology through the implementation of interdisciplinary curricula, continued education, distance learning, and network innovation projects.

Likewise, to overcome scientific illiteracy and misconceptions among the general public, the big topics of today-including climate change, energy resources, nanotechnology, health, and water resourcesshould be used to capture the public's interest, said J.E.E. Baglin (IBM Almaden Research Center, USA). In a society that relies heavily on the use of advanced technologies, a basic knowledge of science is needed to ensure the implementation of intelligent business, government, and legal decisions and to avoid the victimization of the non-technical community in these areas.

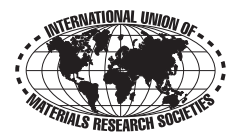

\title{
World Materials Summits for Energy Investigate Economically Viable Materials Research Directions
}

Recognizing the strategic importance of materials research for the world's energy future, the Materials Research Society (MRS), the European MRS (E-MRS), and the Chinese MRS (C-MRS) are collaborating in a joint endeavour to convene science policymakers across international borders to discuss the role of materials for future energy sustainability. The focal point of these collaborations has been two World Materials Summits for Energy, which were conceived not as just another scientific conference but as an event to bring together global leaders in science, technology, and policy to formulate an objective view on economically viable energy materials research directions.

The inaugural summit was held in Lisbon, Portugal on October 4-5, 2007, coinciding with the Portuguese Presidency of the European Union. The scope included reduced $\mathrm{CO}_{2}$ emissions during energy production and use by means of new and innovative materials and processes, solar energy, wind power, biofuels, hydrogen fuel cells, nuclear fission and fusion, and complementary problems in storage, transmission, conservation, and waste utilization. Current projections estimate that energy needs of the world will more than double by the year 2050, a demand that cannot be met by existing technologies. In this context, advanced materials, materials research, and innovation are arguably the most urgent elements to prepare new technologies needed in the not-too-distant future.

At the end of the First Summit, the most important aspects identified were published in the "Lisbon 2007 Declaration on International Cooperation in Materials Research: Key to Meeting Energy Needs and Addressing Climate Change." The meeting concluded that the present pace of research and development was too slow to meet the needs of the world's rapidly growing and increasingly energy-hungry population. Effort was too fragmented and competition between different laboratories and institutions - not to mention governments-hindered progress on complex problems where international cooperation is vital to achieve breakthroughs. It was conveyed that each of the technologies discussed required new and improved materials to increase efficiency and reliability, decrease greenhouse gas emissions, reduce capital costs, and extend operating lifetimes. These conclusions were sometimes in conflict with the views expressed by mainstream media. It was recognized, for example, that energy coming from fossil fuels would continue to dominate the energy landscape for the next several decades, hydrogen fuelled vehicles are far from readiness for the mass market at economically valid conditions, and electric automobiles would be limited to small niche markets by Li-battery stability. Moreover, the availability of critical, rare elements for new technologies to meet global demand is a major concern. It was agreed that solar; further controlled use of nuclear; and in the long term, biological energy sources are eligible technologies in which materials research could bring substantial gains in sustainability attributes. The First Summit was addressed by dignitaries from the European Commission, the European Science Foundation, and prominent scientists from industry as well as government laboratories and academia.

The positive outcome from the First Summit immediately led to planning the 
second Summit of the series, and the Chinese delegation agreed to host in Suzhou. The Second World Materials Summit was organized by C-MRS, E-MRS, and MRS on October 12-15, 2009. This Summit made recommendations on how best to achieve the essential acceleration of development by the worldwide materials science and technology community, and developed a declaration to be presented at the United Nations Climate Change Conference in Copenhagen in December 2009.

The Second Summit concluded that safe nuclear energy is undoubtedly needed in the future energy mix, and it requires reliability and inspectability. Some of the most important challenges in this field are related to fuel cycle issues and public acceptance of nuclear energy: new fuels using minor actinides and a reprocessing cycle that guarantees security, low waste volume, and low toxicity are required. Experts agreed that fusion offers an exciting future, where one long lead-time priority is firstwall materials. Another important issue, which initially arose from the First Summit, was to consider $\mathrm{CO}_{2}$ as a raw material that can be transformed into a chemical fuel. Summit conclusions also included the necessity for further development of solar energy to the terawatt level, which will require exponential growth to serve society in the appropriate time frame. However, the increase in renewable energy will necessitate totally new concepts to ensure the effectiveness of the electrical transmission grid system. Some of these technologies are approaching grid parity (producing power at the same cost as baseload power), but it is not yet clear if they can scale to significant levels based on present limitations in materials, processes, and devices. Public support needs to be gained through education in order to obtain sustained societal adoption of these emerging technologies.

There emerged also significant technical questions related to efficient energy storage. It was conveyed that fuel cell technology is the cleanest and most efficient way of converting chemical energy directly into electrical energy without combustion. Among the possible energy carriers for storage and mobile applications, hydrogen appeared as the ideal one, owing to its energy density. However, several challenges have to be faced for hydrogen production and storage, such as the efficient separation and purification of hydrogen, and the improvement of the quality and stability of the materials used for its production, including catalysts that will allow a decrease in the cost of hydrogen production. From another side, it was recognized that lithium ion batteries (LiB) represent an efficient solution for $\mathrm{CO}_{2}$ abatement in the transportation sector. However, to ensure full safety of LiBs, electrolytes have to be improved and electrodes should be replaced by negative electrode materials with high capacity. Finally, it was clear that bio-energy is renewable and sustainable with costs potentially competitive with fossil sources, including environmental compatibility issues. However, the treatment of cellulosic materials is currently expensive; efficiency for fermentation has to be increased; and a balance must be established between food, fuel, and other land uses since biomass crops should not compromise food supply.

The Second World Materials Summit provided an avenue to create international cooperation on energy-related materials solutions, taking as priorities the relevant sections of the Kyoto resolution about climate change. New concepts, closer collaboration between the fundamental research sector and industry, a new and sustained international interdisciplinary materials research collaboration, and closer cooperation between all countries of the world are necessary for this finality. The Summit also proposed to promote the education of a new generation of international scientists, engineers, and leaders for leveraging materials science and technology for energy research and development and to provide a clear picture of challenges, opportunities, and career paths.

Much work remains as the materials community addresses the critical global energy challenges. The successful collaborations from the first two Summits provide a path forward as MRS, E-MRS and C-MRS have begun planning for the 2011 Materials World Summit in Washington D.C.

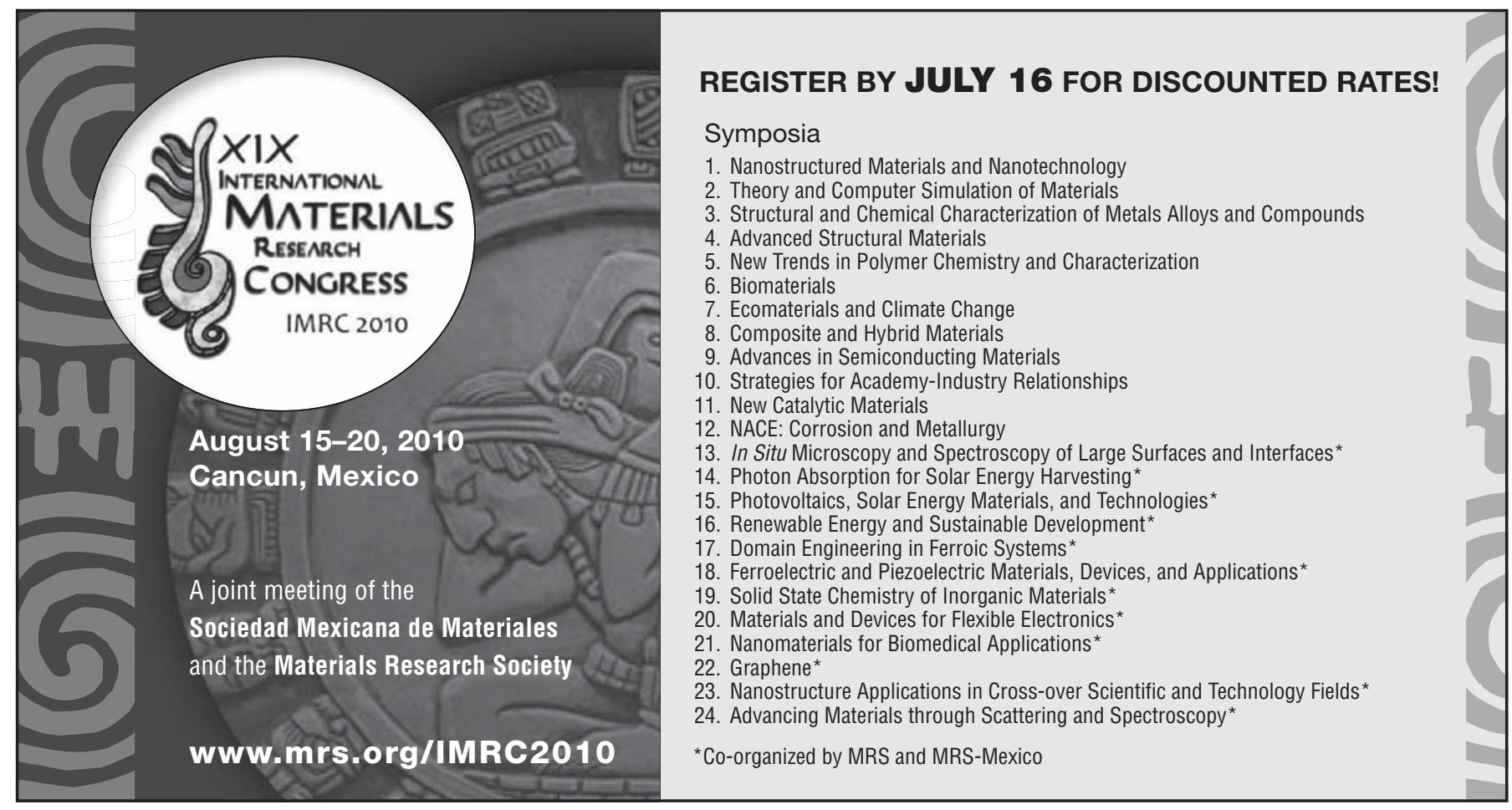

